\title{
Subdirectly irreducible semilattices with endomorphism
}

\author{
Jie Fang (1D \\ School of Mathematics and System Science, Guangdong Polytechnic Normal University, China
}

\begin{abstract}
In this paper we initiate an investigation into the class of meet semilattices endowed with an endomorphism. A consideration of the subdirectly irreducible algebras leads to a description of a subclass of those algebras $(S ; \wedge, k)$ in which $(S ; \wedge)$ is a meet semilattice and $k$ is an endomorphism on $S$ characterised by the property $k \geqslant i d_{S}$. We particularly show that such an algebra is subdirectly irreducible if and only if it is a chain with one of the following forms:
\end{abstract}

(1) $\cdots<a_{j}<a_{j-1}<\cdots<a_{0}$;

(2) $0<\cdots<a_{j}<a_{j-1}<\cdots<a_{0}$

in which $k\left(a_{j}\right)=a_{j-1}$ for $j \geqslant 1, k(0)=0$ and $k\left(a_{0}\right)=a_{0}$.

Mathematics Subject Classification (2020). 06A12, 06A11, 06B10

Keywords. semilattice, endomorphism, subdirectly irreducible

\section{Introduction}

An ordered set $(S ; \leqslant)$ is a meet-semilatice if for any $x, y \in S$, the greatest lower bound $\inf \{x, y\}$ of $x$ and $y$ exists, denoted by $x \wedge y$. A mapping $k: S \rightarrow S$ is said to be endomorphism if $k(x \wedge y)=k(x) \wedge k(y)$ for every $x, y \in S$. For any additional background see, for example, either of the texts Blyth [1] or Grätzer [5].

Throughout what follows, we shall use the terminology $(S ; \wedge)$ to denote a meet- semilattice.

In 1991, Ježek [8] initiated a study of the class of semilattices with an automorphism by characterising its subdirectly irreducible members. In 2004, Jackson [7] introduced a class of closure semilattices. In particular, he gave a representation of semilattices by means of topological Boolean algebras. Furthermore, the other related topics can be found in $[2,6]$.

Here our objective is to initiate an investigation into the class of semilattices $(S ; \wedge)$ endowed with an endomorphism $k$. We shall say that such an algebra $(S ; \wedge, k)$ is an SLEalgebra; namely, an SLE-algebra is an algebra $(S ; \wedge, k)$ of type $\langle 2,1\rangle$ where $(S ; \wedge)$ is a meet-semilattice, and $k$ is an endomorphism on $S$.

In what follows we shall denote by SLE the class of SLE-algebras.

Example 1.1. Every meet-semilattice gives to an SLE-algebra. For example, if $(S ; \wedge)$ is a semilattice, then $\left(S ; \wedge, \operatorname{id}_{S}\right) \in \mathbf{S L E}$.

Email address: jfang@gpnu.edu.cn

Received: 12.09.2021; Accepted: 14.10.2021 
Example 1.2. Let $(S ; \wedge)$ be a meet-semilattice and $a \in S$. If define $k: S \rightarrow S$ by $k(x)=a \wedge x$, then clearly $(S ; \wedge, k) \in \mathbf{S L E}$.

Example 1.3. Consider a semilattice $(S ; \wedge)$ described as the following Hasse diagram:

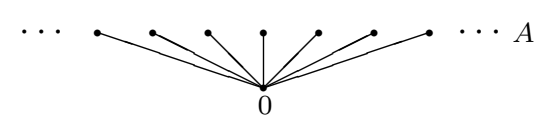

in which $S=A \cup\{0\}$ where $A=\left\{a_{i} \mid i \in \mathbb{N}\right\}$ is an anti-chain, and $0<a_{i}$ for all $i \in \mathbb{N}$. Define $k: S \rightarrow S$ given by $k(0)=0$ and $k\left(a_{i}\right)=a_{i+1}$ for each $i \in \mathbb{N}$. Then $(S ; \wedge, k) \in$ SLE.

Example 1.4. Consider an infinite SLE-algebra $(S ; \wedge, k)$ depicted as follows:

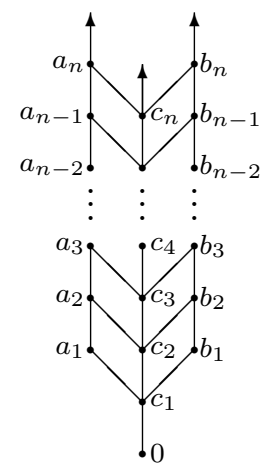

in which $c_{i}=a_{i} \wedge b_{i}(i=1,2, \cdots)$, the endomorphism $k: S \rightarrow S$ is defined by $k(0)=0$ and $k\left(x_{i}\right)=x_{i+1}$ where $x_{i} \in\left\{a_{i}, b_{i}, c_{i}\right\}$.

\section{Congruences}

By a semilattice congruence we shall mean an equivalence relation $\varphi$ on a semilattice $(S ; \wedge)$ satisfying the following condition:

$$
(\forall x, y \in S)(\forall z \in S) \quad(x, y) \in \varphi \Longrightarrow(x \wedge z, y \wedge z) \in \varphi .
$$

As usual we shall denote by $\omega$ and $\iota$ the equality relation and the universal relation, respectively. For $a, b \in S$ with $a \leqslant b$, we denote by $\theta_{S}(a, b)$ the principal semilattice congruence that collapses $a$ and $b$; i.e., the smallest semilattice congruence on $S$ generated by $a$ and $b$.

The following description of the principal semilattice congruences is due to Dean and Oehmke (see [3] or [4]).

Lemma 2.1. [4, Lemma 2.1] If $(S ; \wedge)$ is a semilattice and $a \leqslant b$ in $S$. Then

$$
(x, y) \in \theta_{S}(a, b) \Longleftrightarrow x=y \text { or } x \wedge a=y \wedge a \text { with } x, y \leqslant b .
$$

Furthermore, for arbitrary $a$ and $b$ in $S, \theta_{S}(a, b)=\theta_{S}(a \wedge b, a) \vee \theta_{S}(a \wedge b, b)$.

As a consequence of the above, the following lemma is immediate:

Lemma 2.2. If $(S ; \wedge)$ is a semilattice then the following statements hold:

(1) If $a, b, c \in S$ with $a \leqslant b$ and $a \leqslant c$ then

$$
\theta_{S}(a, b) \wedge \theta_{S}(a, c)=\theta_{S}(a, b \wedge c) .
$$

(2) If $a, b, c, d \in S$ with $a \leqslant b \leqslant c \leqslant d$ then

$$
\theta_{S}(a, b) \wedge \theta_{S}(c, d)=\omega \text {. }
$$


By a congruence on an SLE-algebra $(S ; k)$ we shall mean a semilattice congruence $\vartheta$ that satisfies the condition

$$
(x, y) \in \vartheta \Longrightarrow(k(x), k(y)) \in \vartheta .
$$

In what follows for $a, b \in S$ with $a \leqslant b$, we shall denote by $\theta(a, b)$ the principal congruence on $(S ; k)$ that collapses $a$ and $b$; i.e., the smallest semilattice congruence on $S$ generated by $a$ and $b$

A description of principal congruences on an SLE-algebra $(S ; \wedge, k)$ can be given as follows.

Theorem 2.3. If $(S ; \wedge, k) \in \mathbf{S L E}$ and $a \leqslant b$ in $S$ then we have

$$
\theta(a, b)=\bigvee_{i \geqslant 0} \theta_{S}\left(k^{i}(a), k^{i}(b)\right) .
$$

Proof. Let $\varphi(a, b)$ be the right side of the stated equality. Then clearly $\varphi(a, b)$ is a semilattice congruence that collapses $a$ and $b$. To see that $\varphi(a, b)$ is a SLE-congruence, it suffices to verify that for every $i \geqslant 0$,

$$
(x, y) \in \theta_{S}\left(k^{i}(a), k^{i}(b)\right) \Longrightarrow(k(x), k(y)) \in \theta_{S}\left(k^{i+1}(a), k^{i+1}(b)\right) .
$$

In fact, if $(x, y) \in \theta_{S}\left(k^{i}(a), k^{i}(b)\right)$ then we have either $x=y$ or $x \wedge k^{i}(a)=y \wedge k^{i}(a)$ with $x, y \leqslant k^{i}(b)$, so either $k(x)=k(y)$ or $k(x) \wedge k^{i+1}(a)=k(y) \wedge k^{i+1}(a)$ with $k(x), k(y) \leqslant$ $k^{i+1}(b)$. It then follows that $(k(x), k(y)) \in \theta_{S}\left(k^{i+1}(a), k^{i+1}(b)\right)$. Hence, $\varphi(a, b)$ is a SLEcongruence.

If now $\psi$ is a congruence on $(S ; \wedge, k)$ that collapses $a$ and $b$, then for every $i \geqslant 0$, we have $\left(k^{i}(a), k^{i}(b)\right) \in \psi$, so $\theta_{S}\left(k^{i}(a), k^{i}(b)\right) \leqslant \psi$, and so

$$
\varphi(a, b)=\bigvee_{i \geqslant 0} \theta_{S}\left(k^{i}(a), k^{i}(b)\right) \leqslant \psi .
$$

Consequently, it follows that $\varphi(a, b)=\theta(a, b)$, as our required.

By Theorem 2.3, the following corollary is immediate.

Corollary 2.4. Let $(S ; \wedge, k) \in \mathbf{S L E}$ and $a \leqslant b$ in $S$. Then we have the followings:

(1) If $k^{m+1}(x)=k^{m}(x)$ for some $m \geqslant 0$ where $x=a$ or $x=b$, then

$$
\theta\left(k^{m}(a), k^{m}(b)\right)=\theta_{S}\left(k^{m}(a), k^{m}(b) ;\right.
$$

(2) If $k^{n}(a)=k^{n}(b)$ for some $n \geqslant 1$, then we have

$$
\theta(a, b)=\bigvee_{i=0}^{n-1} \theta_{S}\left(k^{i}(a), k^{i}(b)\right) \text {. }
$$

Here we shall be concerned with those SLE-algebras $(S ; \wedge, k)$ in which $k$ satisfies the property $k \geqslant \operatorname{id}_{S}$. Clearly, if $(S ; \wedge, k)$ is such an algebra then we have

$$
x \leqslant k(x) \leqslant k^{2}(x) \leqslant \cdots \leqslant k^{n}(x) \leqslant \cdots
$$

for every $x \in S$.

Example 2.5. The constructions in Examples 1.1 and 1.4 give the SLE-algebras $(S ; \wedge, k)$ for which $k \geqslant \operatorname{id}_{S}$.

For the later purpose, we require the following basic properties.

Theorem 2.6. Let $(S ; \wedge, k) \in \mathbf{S L E}$ with $k \geqslant \mathrm{id}_{S}$. If $a, b \in S$ with $a \leqslant b$ are such that $k^{n+1}(x)=k^{n}(x)$ for some $n \geqslant 0$ where $x=a$ or $x=b$, then we have the following properties:

(1) $\theta\left(a, k^{n}(a)\right)=\theta_{S}\left(a, k^{n}(a)\right)$;

(2) if $k(b)=b$ then $\theta(a, b)=\theta_{S}(a, b)$;

(3) if $k(a)=a$ then $\theta(a, b)=\theta_{S}\left(a, k^{n}(b)\right)$. 
Proof. (1) Since $k^{i}\left(k^{n}(a)\right)=k^{n}(a)$ for each $i$, we have by Theorem 2.3 that

$$
\theta\left(a, k^{n}(a)\right)=\bigvee_{i \geqslant 0} \theta_{S}\left(k^{i}(a), k^{i}\left(k^{n}(a)\right)\right)=\bigvee_{i=0}^{n} \theta_{S}\left(k^{i}(a), k^{n}(a)\right)
$$

Note that $a \leqslant k^{i}(a)$ for each $i$, then we have $\theta_{S}\left(k^{i}(a), k^{n}(a)\right) \leqslant \theta_{S}\left(a, k^{n}(a)\right)$. Thus it follows that $\theta\left(a, k^{n}(a)\right)=\theta_{S}\left(a, k^{n}(a)\right)$.

(2) If $k(b)=b$ then, since $a \leqslant k^{i}(a)$, we have $\theta_{S}\left(k^{i}(a), b\right) \leqslant \theta_{S}(a, b)$ for each $i$. It follows by Theorem 2.3 that

$$
\theta(a, b)=\bigvee_{i=0}^{n} \theta_{S}\left(k^{i}(a), b\right)=\theta_{S}(a, b)
$$

(3) If $k(a)=a$ then $\theta(a, b)=\bigvee_{i=0}^{n} \theta_{S}\left(a, k^{i}(b)\right)$. Since $k^{i}(b) \leqslant k^{n}(b)$, we have $\theta_{S}\left(a, k^{i}(b)\right) \leqslant$ $\theta_{S}\left(a, k^{n}(b)\right)$ for each $i$. Thus the stated equality holds.

\section{Subdirectly irreducible}

Our main interest here will be in the subdirectly irreducible algebras. We recall (see [1] or [5]) that an algebra $\mathcal{A}$ is said to be subdirectly irreducible if for any family $\left\{\vartheta_{i} \mid i \in I\right\}$ of congruences on $\mathcal{A}, \bigwedge_{i \in I} \vartheta_{i}=\omega$ implies $\vartheta_{i}=\omega$ for some $i$; equivalently, there exists a smallest nontrival congruence $\vartheta$ on $\mathcal{A}$ such that $\varphi \geqslant \vartheta$ for every congruence $\varphi \neq \omega$ on $\mathcal{A}$.

The following result will play an important rôle.

Theorem 3.1. Let $(S ; \wedge, k) \in \mathbf{S L E}$ with $k \geqslant \operatorname{id}_{S}$. If $S$ is subdirectly irreducible algebra then for every $a \in S$ there exists some $n \geqslant 0$ such that $k^{n+1}(a)=k^{n}(a)$.

Proof. Let $S$ be subdirectly irreducible, and suppose, by the way of contradiction, that there exists some $a \in S$ such that $k^{n+1}(a) \neq k^{n}(a)$ for all $n$. Then we have

$$
a<k(a)<k^{2}(a)<\cdots<k^{n}(a)<\cdots .
$$

Thus for all $i \geqslant 0, \theta\left(k^{i}(a), k^{i+1}(a)\right)>\omega$. If

$$
\text { (घ) } \quad(x, y) \in \bigwedge_{i \geqslant 0} \theta\left(k^{i}(a), k^{i+1}(a)\right)
$$

then for each $i$, we have

$$
(x, y) \in \theta\left(k^{i}(a), k^{i+1}(a)\right)=\bigvee_{j \geqslant i} \theta_{S}\left(k^{j}(a), k^{j+1}(a)\right) .
$$

It follows that there exists some $r \geqslant i$ such that

$$
(x, y) \in \bigvee_{j=i}^{r-1} \theta_{S}\left(k^{j}(a), k^{j+1}(a)\right)=\theta_{S}\left(k^{i}(a), k^{r}(a)\right)
$$

By ( $(4)$, we have also $(x, y) \in \theta\left(k^{r}(a), k^{r+1}(a)\right)$, and similarly, $(x, y) \in \theta_{S}\left(k^{r}(a), k^{t}(a)\right)$ for some $t \geqslant r$. Thus

$$
(x, y) \in \theta_{S}\left(k^{i}(a), k^{r}(a)\right) \wedge \theta_{S}\left(k^{r}(a), k^{t}(a)\right)=\omega
$$

whence we obtain $x=y$. It therefore follows that

$$
\bigwedge_{i \geqslant 0} \theta\left(k^{i}(a), k^{i+1}(a)\right)=\omega .
$$

This contradicts the assumption of the subdirectly irreducibility. Hence, there is some $n \geqslant 0$ such that $k^{n+1}(a)=k^{n}(a)$. 
Example 3.2. Consider an infinite SLE-algebra $(S ; \wedge, k)$ depicted as follows:

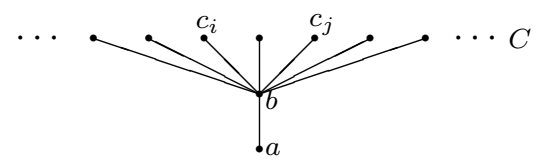

in which $S=\{a, b\} \cup C$ where $C=\left\{c_{i} \mid i \in \mathbb{N}\right\}$ is an anti-chain, and $a<b<c_{i}, c_{i} \wedge c_{j}=b$ for every $i, j \in \mathbb{N}(i \neq j)$, and the endomorphism $k: S \rightarrow S$ is given by $k(a)=k(b)=b$ and $k\left(c_{i}\right)=c_{i}$ for each $i \in \mathbb{N}$. Then $k \geqslant \operatorname{id}_{S}$ and clearly $x \leqslant k(x)=k^{2}(x)$ for every $x \in S$. Observe that for any $i, j$ with $i \neq j$,

$$
\begin{aligned}
\theta\left(b, c_{i}\right) \wedge \theta\left(b, c_{j}\right) & =\theta_{S}\left(b, c_{i}\right) \wedge \theta_{S}\left(b, c_{j}\right) \\
& =\theta_{S}\left(b, c_{i} \wedge c_{j}\right) \\
& =\theta_{S}(b, b) \\
& =\omega .
\end{aligned}
$$

Then we see that $(S ; \wedge, k)$ is not subdirectly irreducible.

For an SLE-algebra $(S ; \wedge, k)$, an element $x \in S$ is said to be fixed point if $k(x)=x$. We shall denote by Fix $S$ the set of fixed points of $S$.

Theorem 3.3. Let $(S ; \wedge, k) \in \mathbf{S L E}$ with $k \geqslant \operatorname{id}_{S}$. If $S$ is subdirectly irreducible algebra then Fix $S$ is a singleton or an 2-element chain.

Proof. By Theorem 3.1, we have clearly that $\mid$ Fix $S \mid \geqslant 1$. Observe now that Fix $S$ is a chain. In fact, if $x, y \in$ Fix $S$, then we have by Theorem 2.3 and Lemma 2.2 that

$$
\begin{aligned}
\theta(x \wedge y, x) \wedge \theta(x \wedge y, y) & =\theta_{S}(x \wedge y, x) \wedge \theta_{S}(x \wedge y, y) \\
& =\theta_{S}(x \wedge y, x \wedge y) \\
& =\omega .
\end{aligned}
$$

It follows by the subdirectly irreducibility that $\theta(x \wedge y, x)=\omega$ or $\theta(x \wedge y, y)=\omega$, from which it follows that $x \wedge y=x$ or $x \wedge y=y$, whence $x \leqslant y$ or $y \leqslant x$. Hence, Fix $S$ is a chain.

If now $\mid$ Fix $S \mid \geqslant 3$, then there exist $x, y, z \in$ Fix $S$ with $x<y<z$. It then follows the contradiction that

$$
\theta(x, y) \wedge \theta(y, z)=\theta_{S}(x, y) \wedge \theta_{S}(y, z)=\omega .
$$

Therefore, Fix $S$ must be a singleton or an 2-element chain.

Corollary 3.4. A meet semilattice $(S ; \wedge)$ is subdirectly irreducible if and only if it is a singleton or an 2-element chain.

Proof. Note that $(S ; \wedge)$ can be regarded as $\left(S ; \wedge, \mathrm{id}_{S}\right)$, then Fix $S=S$. Thus by Theorem 3.3 , the conclusion is clear.

In what follows for an ordered set $S$ and $a, b \in S$, we shall write $a \nVdash b$ to denote that $a$ and $b$ are comparable (in the sense that $a \leqslant b$ or $b \leqslant a$ ), and write $a \| b$ to denote that $a$ and $b$ are incomparable (in the sense that $a \nless b$ and $b \nless a$ ).

Theorem 3.5. Let $(S ; \wedge, k) \in \mathbf{S L E}$ with $k \geqslant \operatorname{id}_{S}$. If $S$ is subdirectly irreducible then $S$ is a chain.

Proof. Suppose that $S$ is subdirectly irreducible, and let $a, b \in S$. Then by Theorems 3.1 and $3.3, k^{n}(a), k^{n}(b) \in$ Fix $S$ with $k^{n}(a) \nVdash k^{n}(b)$ for some $n \geqslant 0$. We may assume that $k^{n}(a) \leqslant k^{n}(b)$. Let $c=a \wedge b$, then $k^{n}(c)=k^{n}(a)$. If $k(a)=k(b)$ then $\theta(c, a) \wedge \theta(c, b)=$ 
$\theta_{S}(c, a) \wedge \theta_{S}(c, b)=\omega$, from which it follows by subdirectly irreducibility that $c=a$ or $c=b$, whence $a \leqslant b$ or $b \leqslant a$. Assume now that $k(a) \neq k(b)$. If $k^{n}(a) \neq k^{n}(b)$, then since

$$
\begin{aligned}
& \theta\left(k^{n-1}(c), k^{n-1}(a)\right) \wedge \theta\left(k^{n}(a), k^{n}(b)\right) \\
& =\theta_{S}\left(k^{n-1}(c), k^{n-1}(a)\right) \wedge \theta_{S}\left(k^{n}(a), k^{n}(b)\right) \\
& =\omega
\end{aligned}
$$

it follows by the subdirectly irreducibility that $k^{n-1}(c)=k^{n-1}(a)$. Then $\theta\left(k^{n-2}(c), k^{n-2}(a)\right)$ $=\theta_{S}\left(k^{n-2}(c), k^{n-2}(a)\right)$. Similar to the above, we can obtain $k^{n-2}(c)=k^{n-2}(a)$. Continuing in this way, we obtain $c=a$, whence $a \leqslant b$. Similarly, if $k^{n}(b)<k^{n}(a)$, then we can obtain that $b \leqslant a$.

If, on the other hand, $k^{n}(a)=k^{n}(b)$, then since $k(a) \neq k(b)$, we have some $m$ with $1 \leqslant m \leqslant n-1$ such that $k^{m}(a) \neq k^{m}(b)$ and $k^{m+1}(a)=k^{m+1}(b)$. Since

$$
\begin{aligned}
& \theta\left(k^{m}(c), k^{m}(a)\right) \wedge \theta\left(k^{m}(c), k^{m}(b)\right) \\
& =\theta_{S}\left(k^{m}(c), k^{m}(a)\right) \wedge \theta_{S}\left(k^{m}(c), k^{m}(b)\right) \\
& =\theta_{S}\left(k^{m}(c), k^{m}(a) \wedge k^{m}(b)\right) \\
& =\omega
\end{aligned}
$$

it follows by the subdirectly irreducibility that $k^{m}(c)=k^{m}(a)$ or $k^{m}(c)=k^{m}(b)$. Thus we have $k^{m}(a)<k^{m}(b)$ or $k^{m}(b)<k^{m}(a)$. By a similar argument as above we also can show that $a \nVdash b$.

Therefore, we see that $S$ is a chain.

Example 3.6. Consider a finite chain $S$ described as follows:

$$
a_{1}<b_{1}<a_{2}<b_{2}<\cdots<a_{n-1}<b_{n-1}<a_{n}=b_{n} .
$$

If define $k: S \rightarrow S$ by $k\left(a_{i}\right)=a_{i+1}, k\left(b_{i}\right)=b_{i+1}$ for $i=1,2, \cdots, n-1$, and $k\left(a_{n}\right)=a_{n}$, then $(S ; \wedge, k) \in \mathbf{S L E}$ with $k \geqslant \operatorname{id}_{S}$. Since

$$
\theta\left(a_{n-1}, b_{n-1}\right) \wedge \theta\left(b_{n-1}, b_{n}\right)=\theta_{S}\left(a_{n-1}, b_{n-1}\right) \wedge \theta_{S}\left(b_{n-1}, b_{n}\right)=\omega,
$$

we see that $S$ is clearly not subdirectly irreducible.

In what follows for $a<b$ in $S$, if the interval $[a, b]=\{x \in S \mid a \leqslant x \leqslant b\}$ is precisely the 2-element set $\{a, b\}$, then we say that $b$ covers $a$ or $a$ is covered by $b$, denoted by $a \prec b$. We shall write $a \preceq b$ to denote that $a=b$ or $a \prec b$.

Theorem 3.7. Let $(S ; \wedge, k) \in \mathbf{S L E}$ with $k \geqslant \mathrm{id}_{S}$. If $S$ is subdirectly irreducible and $a \in S$, then we have $k^{i-1}(a) \preceq k^{i}(a)$ for every $i \geqslant 1$.

Proof. Let $a \in S$ and suppose that $b \in S$ is such that $k^{i-1}(a) \leqslant b \leqslant k^{i}(a)$ for some $i \geqslant 1$. If $k(a)=a$ then there is nothing to do, and if $k(b)=b$, then we have clearly $b=k^{i}(a)$. We may assume that $k(a) \neq a$ and $k(b) \neq b$. Then by Theorem 3.1, we have $m \geqslant 1$ and $n \geqslant 1$ such that

$$
\begin{aligned}
& a<k(a)<\cdots<k^{n-1}(a)<k^{n}(a)=k^{n+1}(a) ; \\
& b<k(b)<\cdots<k^{m-1}(b)<k^{m}(b)=k^{m+1}(b) .
\end{aligned}
$$

Let $t=n-i+1$ then $k^{t}(b)=k^{n}(a) \in \operatorname{Fix} S$. Thus we have $t \geqslant m$. Write $c=k^{i-1}(a)$ then $k^{t}(c)=k^{n}(a) \in \operatorname{Fix} S$, and since $c \leqslant b \leqslant k(c)$, we have $k^{m+1}(c)=k^{m}(b) \in \operatorname{Fix} S$, there follows that $m+1 \geqslant t \geqslant m$. Hence, there are two possibilities to consider:

(1) If $t=m+1$ then $k^{m}(c) \notin \operatorname{Fix} S$. Thus we have $k^{m}(c) \neq k^{m}(b)$. Since by Corollary 2.4 ,

$$
\begin{aligned}
& \theta\left(k^{m-1}(b), k^{m}(c)\right) \wedge \theta\left(k^{m}(c), k^{m}(b)\right) \\
& =\theta_{S}\left(k^{m-1}(b), k^{m}(c)\right) \wedge \theta_{S}\left(k^{m}(c), k^{m}(b)\right) \\
& =\omega
\end{aligned}
$$


it follows by the subdirectly irreducibility that $k^{m-1}(b)=k^{m}(c)$. Then $\theta\left(k^{m-2}(b), k^{m-1}(c)\right)$ $=\theta_{S}\left(k^{m-2}(b), k^{m-1}(c)\right)$. Similar to the above, we can obtain $k^{m-2}(b)=k^{m-1}(c)$. Continuing in this way, we have $b=k(c)=k^{i}(a)$.

(2) If $t=m$ then $k^{m}(c)=k^{m}(b)$. Since $k^{m-1}(b)<k^{m}(b)=k^{m+1}(b)$, we can obtain by a similar argument as in (1) that $b=c=k^{i-1}(a)$.

Therefore, we see that $k^{i-1}(a) \preceq k^{i}(a)$.

With combination of Theorems 3.1, 3.3, 3.5 and 3.7, we now can give our main result as follows.

Theorem 3.8. If $(S ; \wedge, k)$ with $k \geqslant \operatorname{id}_{S}$ is an $\mathrm{SLE-algebra} \mathrm{then} S$ is subdirectly irreducible if and only if it is a chain with one of the following forms:

(1) $\cdots<a_{j}<a_{j-1}<\cdots<a_{0}$;

(2) $0<\cdots<a_{j}<a_{j-1}<\cdots<a_{0}$

in which $k\left(a_{j}\right)=a_{j-1}$ for $j \geqslant 1, k(0)=0$ and $k\left(a_{0}\right)=a_{0}$.

Proof. $(\Rightarrow$ :) Suppose that $S$ is subdirectly irreducible. Then by Theorem $3.5, S$ is a chain. If $|S|=1$; i.e., $S=\{0\}$, then we have clearly $k(0)=0$. If $|S|=2$ then $S$ is clearly an 2-element chain as $0=k(0)<a_{0}=k\left(a_{0}\right)$ or $a_{1}<a_{0}$ with $k\left(a_{1}\right)=k\left(a_{0}\right)=a_{0}$.

We now may assume that $|S| \geqslant 3$. Then since $\mid$ Fix $S \mid \leqslant 2$, there exists $a \in S$ with $k(a) \neq a$, and by Theorem 3.1 we have some $n \geqslant 1$ such that $k^{n}(a) \in \operatorname{Fix} S$ but $k^{n-1}(a) \notin$ Fix $S$. It then follows by Theorem 3.7 that

$$
\text { (†) } a \prec k(a) \prec \cdots \prec k^{n-1}(a) \prec k^{n}(a) .
$$

We shall show as follows that if $b \in \operatorname{Fix} S$ with $b \neq k^{n}(a)$ then $b=0$, the bottom element of $S$. Observe first that $b<a$. In fact, if $b \geqslant a$ then $b>k^{n}(a)$, there follows the contradiction that

$$
\begin{aligned}
& \theta\left(k^{n-1}(a), k^{n}(a)\right) \wedge \theta\left(k^{n}(a), b\right) \\
& =\theta_{S}\left(k^{n-1}(a), k^{n}(a)\right) \wedge \theta_{S}\left(k^{n}(a), b\right) \\
& =\omega .
\end{aligned}
$$

Thus we have $b<a$. Now for $x \in S$, if $x<b$, then it follows by Theorem 2.6 the contradiction that

$$
\theta(x, b) \wedge \theta\left(a, k^{n}(a)\right)=\theta_{S}(x, b) \wedge \theta_{S}\left(a, k^{n}(a)\right)=\omega .
$$

Hence we have $x \geqslant b$, whence $b=0$.

To see that $S$ is of one of the stated forms, it suffices to show that for $x \in S$, if $x \notin$ Fix $S$ and $x \neq k^{i}(a)$ for each $i$, then $k^{j}(x)=a$ for some $j$. By Theorems 3.1, 3.3 and 3.7, we have some $m \geqslant 1$ such that

$$
(\ddagger) \quad x \prec k(x) \prec \cdots \prec k^{m-1}(x) \prec k^{m}(x)=k^{n}(a) .
$$

By (†) we have $x \notin\left[a, k^{n}(a)\right]$. It follows that $x \ngtr a$, so $x<a<k^{n}(a)=k^{m}(x)$. Thus we obtain by $(\ddagger)$ that $a=k^{j}(x)$ for some $j \leqslant m$.

Therefore, if $\mid$ Fix $S \mid=1$, say Fix $S=\left\{k^{n}(a)\right\}$, then $S$ is of the form (1); and if $\mid$ Fix $S \mid=2$, say Fix $S=\left\{b, k^{n}(a)\right\}$, then $S$ is of the form (2).

$(\Leftarrow:)$ Suppose that $S$ is one of the stated forms. If $|S| \leqslant 2$ then it is clearly subdirectly irreducible. We may assume that $|S| \geqslant 3$, and let $\varphi \neq \omega$ be a congruence on $S$. In the form (2), we see $\theta\left(0, a_{i}\right)=\theta\left(0, a_{0}\right)=\iota$ for every $i$. Thus in the either cases, we have $i, j$ with $j>i>0$ such that $\left(a_{j}, a_{i}\right) \in \varphi$, then $\left(a_{j-i}, a_{0}\right)=\left(k^{i}\left(a_{j}\right), k^{i}\left(a_{i}\right)\right) \in \varphi$, and then, we have

$$
\left(a_{1}, a_{0}\right) \in \theta_{S}\left(a_{j-i}, a_{0}\right) \leqslant \theta\left(a_{j-i}, a_{0}\right) \leqslant \varphi
$$

whence $\theta\left(a_{1}, a_{0}\right) \leqslant \varphi$. Hence, it follows that $\theta\left(a_{1}, a_{0}\right)$ is the smallest nontrivial congruence on $S$, and consequently, $S$ is subdirectly irreducible. 
By Theorem 3.8, the following corollary is clear.

Corollary 3.9. Let $(S ; \wedge, k) \in \mathbf{S L E}$ with $k \geqslant \mathrm{id}_{S}$. If $S$ is finite, then $S$ is subdirectly irreducible if and only if it is a chain with one of the following forms:

(1) $a_{m}<a_{m-1}<\cdots<a_{0}$;

(2) $0<a_{m}<a_{m-1}<\cdots<a_{0}$

in which $k\left(a_{j}\right)=a_{j-1}$ for $m \geqslant j \geqslant 1, k(0)=0, k\left(a_{0}\right)=a_{0}$.

Acknowledgment. The author is indebted to the referee for the valuable comments.

\section{References}

[1] T.S. Blyth, Lattices and Ordered Algebraic Structures, Springer-Verlag, London, 2005.

[2] I. Chajda, Congruences on semilattices with section antitone involutions, Discuss. Math. Gen. Algebra Appl. 30 (2), 207-215, 2010.

[3] R.A. Dean and R.H. Ochmke, Idempotent semigroups with distributive right congruence lattices, Pacific J. Math. 14, 1187-1209, 1964.

[4] Jie Fang and Zhongju Sun, Semilattices with the strong endomorphism kernel property, Algebra Universalis, 70 (4), 393-401, 2013.

[5] G. Grätzer, General Lattice Theory, 2nd edn, Birkhäuser, Basel, 1998.

[6] J. Hyndman, J.B. Nation and J. Nishida, Congruence lattices of semilattices with operations, Studia Logica, 104 (2), 305-316, 2016.

[7] Marcel Jackson, Semilattices with closure, Algebra Universalis, 52 (1), 1-37, 2004.

[8] J. Ježek, Subdirectly irreducible semilattices with an automorphism, Semigroup Forum, 43 (2), 178-186, 1991. 